\title{
A PHOTOLUMINESCENCE STUDY IN PbS-EuS SUPERLATTICES
}

L. KowalczyK, J. Sadowski, R.R. GalazzKa

Institute of Physics, Polish Academy of Sciences

Al. Lotników 32/46, 02-668 Warszawa, Poland

\section{A. STAchow-Wójcik}

Institute of Experimental Physics, Warsaw University

Hoża 69, 00-681 Warszawa, Poland

A.Yu. SiPatov, V.V. Volobuev

Kharkov State Technical University, Kharkov, Ukraine

\section{V.A. SMIRNov}

A.F. Ioffe Physical-Technical Institute, St. Petersburg, Russia

AND V.K. DugaEV

Institute of Material Science Problems, Ukrainian Academy of Sciences

Chernivtsy, Ukraine

Investigations of the photoluminescence of $\mathrm{PbS}-\mathrm{EuS}$ superlattices deposited on (111) $\mathrm{BaF}_{2}$ substrates are presented. Quantum-size and deformation effects in photoluminescence spectra are observed. The strain-induced gap shift and valence-band offset is determined from experimental results. A strong stimulated photoluminescence with relatively low threshold was observed. It was found that the photocarriers generated in EuS barrier strongly affect the population of $\mathrm{PbS}$ subbands.

PACS numbers: $75.50 . P p$

\section{Introduction}

Low-dimensional structures on the base of IV-VI narrow-gap semiconductors with europium chalcogenides barrier are of great importance. As is commonly believed they are promising materials for infrared detectors and semiconducting lasers [1]. Therefore, the following important problems arose: understanding the heterojunction energy diagram and deformation shifts in strained heterostructures. 
The present work deals with photoluminescence study of $\mathrm{PbS}-\mathrm{EuS}$ superlattices at liquid helium temperature. So far, the photoluminescence spectra of $\mathrm{PbS}-\mathrm{EuS}$ superlattices [2-4] were described utilising calculations based on a model energy band diagram in which a potential barrier in the valence band was assumed to be equal to zero. In our work, from a comparison of the experimentally determined interband transition energies with the calculated values on several samples the valence-band offset is determined as $\Delta E_{\mathrm{v}}=0.1 \mathrm{eV}$.

\section{Experiment}

A batch of superlattice samples was grown on (111) $\mathrm{BaF}_{2}$ substrates. The thickness of the $\mathrm{PbS}$ well varied from sample to sample within the range $70-175 \AA$ and the thickness of barrier with the range of 6-80 $\AA$. The number of periods was 10. Photoluminescence was excited by YAG:Nd laser pulses of $1.16 \mathrm{eV}$ photon energy or pulses of $2.33 \mathrm{eV}$ photon energy. The pulse duration was 6 ns. The measurements were carried out at liquid helium temperature.

\section{Results and discussion}

Typical photoluminescence spectra of PbS-EuS superlattices for three different intensities of excitation are presented in Fig. 1. The spectra are attributed to transitions between $\mathrm{PbS}$ well states. The arrows mark the calculated energetical positions for the onset of the allowed optical transitions (equal subband numbers).

The superscripts $\|$ (parallel) and $\bigvee$ (antiparallel) refer to both valley groups (one straight and three oblique valleys) in the Brillouin zone which have to be distinguished for (111)-oriented samples. Two groups of carriers are formed and they have different effective masses along the quantization direction. The electron and hole levels in PbS superlattices were calculated by solving the Schrödinger equation in the envelope function approximation. For numerical calculations the transfer matrix method was applied [5]. The dependence of the effective mass on energy according to the Kane band structure was taken into account. For the band edge masses of the straight and oblique valley carriers we took the formula presented in Ref. [4].

The dependence of the low-energy edge of the photoluminescence spectra of $\mathrm{PbS}-\mathrm{EuS}$ superlattices on the thickness of the $\mathrm{PbS}$ well is presented in Fig. 2. The curves denoted $E_{1}$ and $E_{2}$ represent the calculated values of transition energy for the first and second size-quantization subbands, respectively. Solid and dashed lines are for the straight and oblique valleys carriers. The strain-induced gap shift for PbS: $\Delta E_{\mathrm{g}}=+26 \mathrm{meV}$ and valence-band offset: $\Delta E_{\mathrm{v}}=100 \mathrm{meV}$ are determined from the adjustment of the calculated transitions to experimental data.

In the case of some of the PbS-EuS superlattices we observed a strong stimulated photoluminescence. The stimulated emission was characterised by the threshold dependence of photoluminescence intensity on the excitation power. For samples with a thick barrier $(\geq 10 \AA)$ excited by laser pulses of $2.3 \mathrm{eV}$ photon energy we observed a strong stimulated photoluminescence with low threshold connected with optical transitions between second quantization levels of $\mathrm{PbS}$ well (Fig. 3a). 


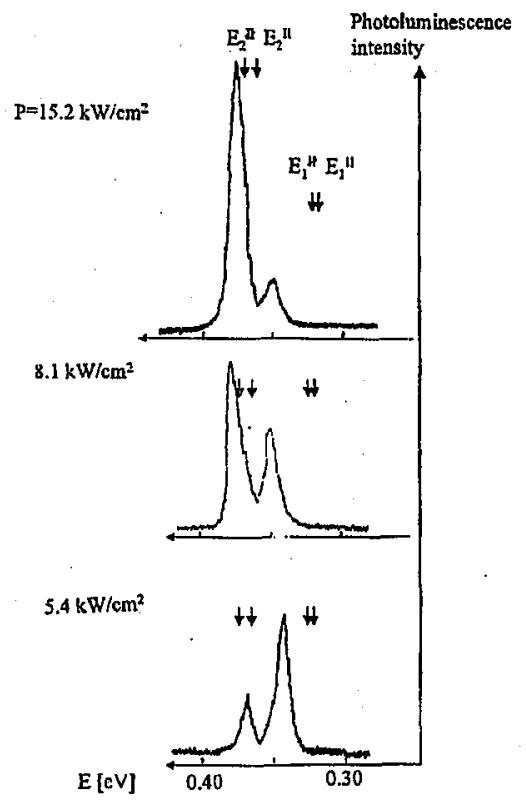

Fig. 1. Photoluminescence spectra from PbS-EuS superlattice ( $170 \AA \mathrm{PbS} / 80 \AA \mathrm{EuS})$ for different excitation power density. The arrows mark the positions calculated for the onset of transitions between well states.

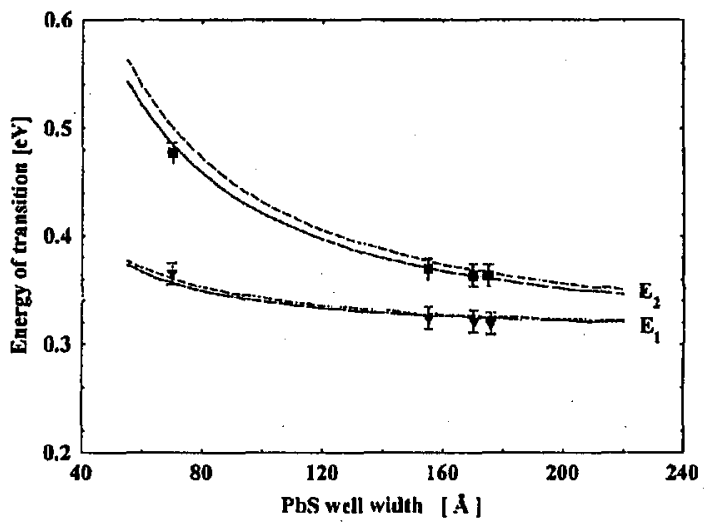

Fig. 2. Dependence of the low edge of the photoluminescence spectra of PbS-EuS superlattices on the thickness of the $\mathrm{PbS}$ well. $E_{1}$ and $E_{2}$ represent the calculated transitions for the first and second size-quantization subbands. Solid and dashed lines are for the straight and oblique valley carriers, respectively.

On the contrary, when the photoluminescence was excited by laser pulses of $1.16 \mathrm{eV}$ photon energy which is absorbed only in $\mathrm{PbS}$ wells (the width of the band gap of $\mathrm{EuS}$ is $1.67 \mathrm{eV}$ ) we were not able to observe stimulated emission in the whole range of the exciting power (Fig. $3 \mathrm{~b}$ ). This result indicates that the photocarri- 

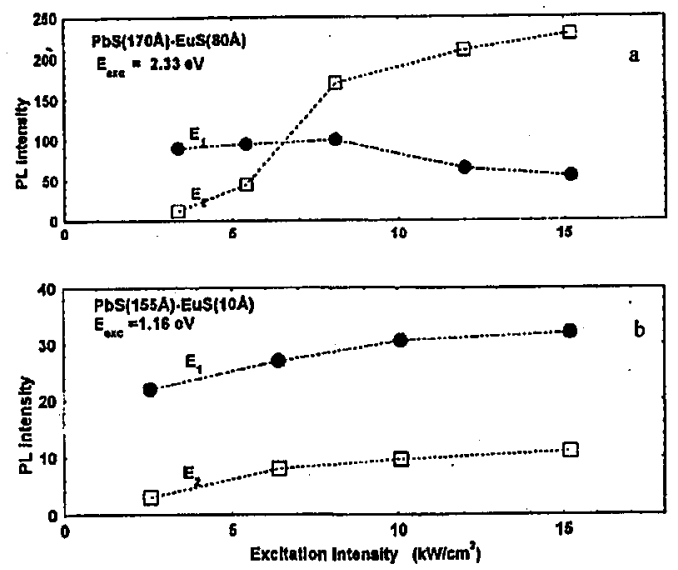

Fig. 3. Photoluminescence intensity (in arb. units) vs. excitation power density. Photon energy of excitation (a) $2.3 \mathrm{eV}$, (b) $1.16 \mathrm{eV}$.

ers generated in EuS barrier are transferred to the narrow-gap $\mathrm{PbS}$ layers that strongly affect the population of $\mathrm{PbS}$ subbands.

In conclusion, the strain-induced gap shift of $\mathrm{PbS}$ and valence-band offset were determined from photoluminescence study of $\mathrm{PbS}-\mathrm{EuS}$ superlattices. The stimulated emission is strongly correlated by pumping the $\mathrm{PbS}$ layers by photocarriers transferred from the barriers.

\section{Acknowledgments}

The authors are indebted to Dr. T. Story and Dr. W. Dobrowolski for many helpful discussions. This work was supported in part by the Committee for Scientific Research (Poland) through grant No. 2P03B10912.

\section{References}

[1] D.L. Partin, IEEE J. Quant. Electron. 24, 1716 (1998).

[2] I.V. Kolesnikov, V.A. Litvinov, A.Yu. Sipatov, A.I. Fedorenko, A.E. Yunovich, Zh. Eksp. Teor. Fiz. 94, 239 (1988).

[3] I.V. Kolesnikov, A.Yu. Sipatov, Fiz. Tekh. Poluprovodn. 23, 954 (1989).

[4] K.H. Herman, J. Anth, K.P. Molman, J.W. Tomm, H. Botner, A. Lambrecht, M. Tacke, I.V. Kolesnikov, A.E. Yunovich, A.I. Federenko, O.A. Mironov, A.Yu. Sipatov, Semicond. Sci. Technol. 8, S176 (1993).

[5] B. Jonsson, S.T. Eng, IEEE J. Quant. Electron. 26, 2052 (1990). 